\title{
三种豆科绿肥作物茎和叶角质层蜡质化学组成分析
}

栗扬 姚露花 郭 欣 赵 晓 黄 蕾 王登科张学风
肖前林 杨瑞吉 郭彦军

西南大学农学与生物科技学院, 重庆 400716

摘 要: 植物角质层蜡质是一类覆盖于植物表层的疏水有机化合物, 在保护植物免受生物与非生物逆境胁迫中发挥 着重要作用。为了更好地了解和认识角质层蜡质在夏季绿肥作物抗逆性中的作用, 选择柽麻(Crotalaria juncea)、田 菁(Sesbania cannabina)和竹豆(Phaseolus calcaratus) 3 种夏季豆科绿肥作物, 鉴定茎和叶蜡质组分, 并分析蜡质总量、 各组分含量及碳链分布特征。共鉴定出 8 类化合物, 包括脂肪酸、初级醇、醛、烷烃、烷基酯、二醇、萜类和固醇 类化合物, 其中前 4 种以同系物形式存在且为所有植物茎和叶共有成分 (柽麻茎中未检出脂肪酸), 说明烷合成和醇合 成途径是主要的 2 种蜡质合成途径。田菁茎中鉴定出二醇化合物, 其结构初步解析为 1,18-30 烷醇和 1,16-30 烷醇。3 种绿肥作物茎和叶蜡质总量存在显著种间及部位差异, 其中柽麻茎蜡质总含量为 $16.33 \mu \mathrm{g} \mathrm{cm}{ }^{-2}$, 显著高于田菁茎 $\left(6.45 \mu \mathrm{g} \mathrm{cm}^{-2}\right)$ 和竹豆茎 $\left(0.72 \mu \mathrm{g} \mathrm{cm}^{-2}\right)$ 。就茎和叶比较, 柽麻茎显著高于叶片, 其他 2 种植物茎和叶之间无显著差异。 柽麻茎蜡质中，烷烃为优势成分，占蜡质总量的 $57.38 \%$; 叶片以初级醇为优势成分，占蜡质总量的 $50.12 \%$ 。田菁茎、 叶蜡质中的优势成分均为初级醇, 分别占总蜡质的 $30.12 \%$ 和 $71.21 \%$ 。竹豆茎、叶蜡质中的优势成分均为烷烃, 分别 占总蜡质的 $40.79 \%$ 和 $39.27 \%$ 。各组分优势化合物的碳链长度在不同物种、不同部位也存在一定差异, 说明参与蜡质 合成的基因在物种、器官间有所不同。这些结果为今后从分子水平上揭示角质层蜡质参与夏季绿肥作物抗逆机制提 供了理论基础。

关键词：角质层蜡质; 绿肥作物; 柽麻; 田菁; 竹亘

\section{Chemical compositions of cuticular waxes on stems and leaves of three legume green manure crops}

LI Yang, YAO Lu-Hua, GUO Xin, ZHAO Xiao, HUANG Lei, WANG Deng-Ke, ZHANG Xue-Feng, XIAO Qian-Lin, YANG Rui-Ji, and GUO Yan-Jun*

College of Agronomy and Biotechnology, Southwest University, Chongqing 400716, China

\begin{abstract}
Cuticular waxes are hydrophobic compounds covering plant surfaces and play vital roles in protecting plants from various biotic and abiotic stresses. However, less is known as related to the cuticular wax on leaves or stems of summer legume green manures. In the current study, stems and leaves from three summer legume green manure crops grown in the fields were sampled, including Crotalaria juncea, Sesbania cannabina, and Phaseolus calcaratus Roxbwere. Using gas chromatography-mass spectrometer (GC-MS), we identified most of the chemical compounds on these plant species. In total, eight classes of wax compounds were identified, including fatty acids, primary alcohols, aldehydes, alkanes, alkyl esters, diols, terpenoids and sterols. Among these compounds, fatty acids, primary alcohols, aldehydes and alkanes with serial homologs could be observed in stems and leaves of all tested plant species excepting fatty acids in leaves of $C$. juncea, with their weight proportions accounting for more than $60 \%$ in total wax. This suggested that alkane forming and alcohol forming pathways were two major biosynthesis pathways in these plants. In stems of $S$. cannabina, two compounds were identified as diols with the functional hydroxyl group
\end{abstract}

本研究由国家自然科学基金项目(31670407)和中央高校基本科研业务费(XDJK2018B021)资助。

This study was supported by the National Natural Science Foundation of China (31670407) and the Fundamental Research Funds for the Central Universities (XDJK2018B021).

*通信作者(Corresponding author): 郭彦军, E-mail: qhgyj@126.com

第一作者联系方式: E-mail: 286923599@qq.com

Received (收稿日期): 2019-03-24; Accepted (接受日期): 2019-05-12; Published online (网络出版日期): 2019-07-22.

URL: http://kns.cnki.net/kcms/detail/11.1809.S.20190719.1721.008.html 
located at 1,18 and 1,16 positions. Using gas chromatography with flam ionized detector (GC/FID), we quantified the total wax and wax compounds. Total wax coverage differed among three plant species and between two organs. The total stem wax coverage was the highest in $C$. juncea $\left(16.33 \mu \mathrm{g} \mathrm{cm}^{-2}\right)$, followed by $S$. cannabina $\left(6.45 \mu \mathrm{g} \mathrm{cm}^{-2}\right)$ and . calcaratus $\left(0.72 \mu \mathrm{g} \mathrm{cm}{ }^{-2}\right)$. The total wax coverage on stems of $C$. juncea was significantly higher than that on leaves, whereas no significant difference in total wax coverage between stems and leaves was observed in $S$. cannabina and $P$. calcaratus. The predominant wax class differed in plant species and organs. For C. juncea, alkanes were the predominant composition in stems accounting for $57.38 \%$ of total wax, whereas primary alcohols were the predominant composition in leaves accounting for $50.12 \%$. For $S$. cannabina, both stems and leaves were dominated by primary alcohols, accounting for $30.12 \%$ and $71.21 \%$ of total wax, respectively. For P. calcaratus, both stems and leaves were dominated by alkanes, accounting for $40.79 \%$ and $39.27 \%$ of total wax, respectively. We further analyzed the chain length distributions of the wax classes in stems and leaves. Generally, fatty acids, primary alcohols and aldehydes were consisted of serials of even carbon number homologs, whereas alkanes were consisted of both even- and odd-carbon number homologs with odd number predominance over even number. The predominant compound in each wax class also differed between plant species and organs. The dominant fatty acids on stems and leaves in $C$. juncea, $S$. cannabina, and P. calcaratus were $\mathrm{C}_{30}, \mathrm{C}_{30}$ and $\mathrm{C}_{26}$, and $\mathrm{C}_{28}$, respectively; the dominant primary alcohols were $\mathrm{C}_{28}, \mathrm{C}_{30}$, and $\mathrm{C}_{28}$ and $\mathrm{C}_{32}$ respectively; the dominant aldehydes were $\mathrm{C}_{32}$ and $\mathrm{C}_{30}, \mathrm{C}_{30}$, and $\mathrm{C}_{30}$ and $\mathrm{C}_{28}$, respectively; while the dominant alkanes were $\mathrm{C}_{31}, \mathrm{C}_{29}$, and $\mathrm{C}_{31}$, respectively. The terpenoids identified in current study were $\beta$-amyrin, $\alpha$-amyrin and lupenol. The variations of predominant wax class and wax compound between plant species and organs implied that genes involved in wax biosynthesis might also be different. These results provide basic knowledges in studying the molecular mechanisms of cuticular waxes in legume green manure crops fronting biotic and abiotic stresses.

Keywords: Cuticular wax; green manures; Crotalaria juncea; Phaseolus calcaratus; Sesbania cannabina

绿肥作物种植于主要作物种植间隙，是一类以 改良土壤质量为目的的植物, 其中以豆科绿肥作物 利用较广 ${ }^{[1]}$ 。豆科绿肥作物既可通过生物固氮增加 土壤中的氮素, 也可通过翻埋入土, 以绿肥的形式 改良土壤质量。然而, 相较于粮食作物, 对夏季绿肥 作物基本理化特性的认识尚不足, 相关研究文献也 少, 制约了研究者对这些绿肥作物的开发利用。因此, 了解夏季绿肥作物的抗性生理指标, 可为提高这类 作物的抗逆性、降低田间管理成本提供理论依据。

植物角质层蜡质是植物与外界环境的第一接触 面, 在保护植物免受生物及非生物胁迫的侵害、提 高抗逆性方面发挥着非常重要的作用 ${ }^{[2]}$ 。如干旱胁 迫下，植物叶角质层蜡质含量的增加被认为与提高 植物的抗旱性有关 ${ }^{[3]}$ 。随着紫外线辐射强度的变化, 角质层蜡质晶体结构也随之发生变化, 以通过反射 减少紫外线伤害 ${ }^{[4]}$ 。角质层蜡质中的醛类物质被认 为与病原菌的侵染有关 ${ }^{[5]}$ 。此外, 朱命炜等 ${ }^{[6]}$ 和韦存 虚等 ${ }^{[7]}$ 应用扫描电镜观察发现成熟芦荟 (Aloe vera) 和星星草(Puccinellia tenuiflora)叶表面分布有密集 的瘤状蜡质, 能有效地减少水分散失和增强叶片反 射光辐射的能力。目前的研究结果表明, 植物角质 层蜡质主要由长链脂肪酸及其衍生物组成, 如醛 类、初级醇、酯类、烷烃、次级醇、酮，及支链醇、 支链烷烃、三萜醇、双酮、烯烃、二醇和酮醇等物 质 ${ }^{8]}$ 。植物种类不同, 其所含的角质层蜡质组分也不 同，如油菜 (Brassica napus) 和番茄 (Lycopersicon esculentum) 以烷类物质为优势成分，紫花苜宿
(Medicago sativa)、玉米(Zea mays) 以初级醇为优势成 分 ${ }^{[9]}$, 羊草 (Leymus chinensis) 以二酮为优势成分 ${ }^{[10]}$ 。 蜡质组分的品种差异说明参与角质层蜡质生物合成 及转运的途径也可能存在差异 ${ }^{[11]}$ 。角质层蜡质的合 成，首先在细胞质中由可溶性的脂肪酸合成酶催化 从头合成 $16 \mathrm{C} / 18 \mathrm{C}$ 的饱和脂肪酸, 然后在内质网中 由脂肪酰-CoA延长酶催化 $16 \mathrm{C} / 18 \mathrm{C}$ 的脂肪酸延长产 生不同链长的长链脂肪酸。脂肪酰-CoA延长酶是一 个多酶复合体，包括 $\beta$-酮脂酰-CoA合酶(3-ketoacylCoA synthase, KCS)、 $\beta$ - 酮脂酰-CoA 还原酶 (3-ketoacyl-CoA reductase, $\mathrm{KCR}) 、 \beta$-差脂酰-CoA脱 水酶(3-hydroxacyl-CoA dehydratase, HCD)和反式烯 脂酰-CoA 还原酶 (trans-2,3-enoyl-CoA reductase, $\mathrm{ECR}$ )。基于蜡质组分分析及基因功能验证，目前已 证实, 角质层蜡质生物合成主要包括脱羰基途径(烷 合成途径)、酰基还原途径(初级醇合成途径)和聚酮 合酶途径(polyketide synthase, PKS) ${ }^{[12]}$ 。如在拟南芥 (Arabidopsis thaliana)中已鉴定出参与蜡质合成的 21 个 $K C S$ 基因、参与合成次级醇和酮的 $\mathrm{MAH} 1$ 基因及参

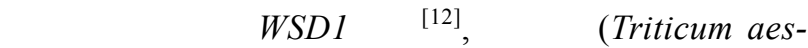
tivum)与大麦 (Hordeum vulgare)中鉴定出参与 $\beta$-二酮 合成的基因 ${ }^{[13]}$ 。挖掘蜡质合成基因已被作为选育抗 逆作物材料的方法之一 ${ }^{[9]}$ 。如 $W X P 1$ 在紫花苜宿中的 超表达，显著增加了烷烃含量，提高了植株的抗旱 性 ${ }^{[13]}$, Os GL1-6在水稻(Oryza sativa)中参与了初级醇 的合成，并可提高水稻抗旱性 ${ }^{[15]}$ 。

柽麻(Crotalaria juncea)、竹豆(Phaseolus cal- 
caratus)、田菁(Sesbania cannabina) 是生产中常用的 几种夏季豆科绿肥作物。柽麻与玉米间作, 可显著 提高玉米地上部产量 ${ }^{[16]}$ 。在松嫩平原盐碱土, 随着 田菁生物量增加, 土壤 $\mathrm{pH}$ 、可溶性盐含量逐年降低, 有机质含量、碱解氮含量逐年升高 ${ }^{[17]}$ 。幼龄果园套 种竹豆绿肥与清耕相比, 可减少径流 $70 \%$ 、泥沙 80 $\%$ 以上, 对改善园地土壤水肥, 促进幼树生长有明 显的促进作用 ${ }^{[18]}$ 。目前尚无这些绿肥作物角质层蜡 质方面的研究报道。鉴于此，本试验从田间采集柽 麻、田菁和竹豆叶片及茎秆, 利用色谱质谱联用仪 鉴定了蜡质组分, 用气相色谱对蜡质组分进行了定 量分析, 并计算了各组分相对含量及碳链分布特征, 为进一步挖掘这些植物的蜡质合成基因, 提高夏季 豆科绿肥作物抗逆性提供理论基础。

\section{1 材料与方法}

\section{1 试验地点}

试验地位于西南大学农学与生物科技学院实验 农场 $\left(29^{\circ} 48^{\prime} \mathrm{N}, 106^{\circ} 24^{\prime} \mathrm{E}\right)$, 属亚热带季风性湿润气候, 年平均气温为 $18.2^{\circ} \mathrm{C}$, 降水量为 $1084.6 \mathrm{~mm}$ 。夏季绿 肥作物生长季 (4 月至 9 月) 月平均气温分别为 $18.9^{\circ} \mathrm{C} 、 22.5^{\circ} \mathrm{C} 、 25.3^{\circ} \mathrm{C} 、 28.6^{\circ} \mathrm{C} 、 28.5^{\circ} \mathrm{C}$ 和 $24.3^{\circ} \mathrm{C}$; 平均降水量分别为 $94.6 、 144.2 、 206.3 、 169.4 、 128.6$ 和 $126.6 \mathrm{~mm}$; 月平均日照时数分别为 $96.8 、 103.8$ 、 $97.2 、 162.5 、 164.3$ 和 $104.7 \mathrm{~h}(1988$ - 2017 年)。供 试土壤为粘质黄壤, $0 \sim 20 \mathrm{~cm}$ 土壤耕层 $\mathrm{pH} 6.37$, 含 有机质 $12.4 \mathrm{~g} \mathrm{~kg}^{-1}$ 、全氮 $0.73 \mathrm{~g} \mathrm{~kg}^{-1}$ 、全磷 $0.12 \mathrm{~g}$ $\mathrm{kg}^{-1}$ 、全钾 $3.32 \mathrm{~g} \mathrm{~kg}^{-1}$ 、碱解氮 $67.4 \mathrm{mg} \mathrm{kg}$ 、速效 磷 $8.6 \mathrm{mg} \mathrm{kg}^{-1}$ 、速效钾 $82.5 \mathrm{mg} \mathrm{kg}^{-1}$ 。

\section{2 绿肥作物}

供试绿肥作物包括柽麻(Crotalaria juncea)、竹 豆 (Phaseolus calcaratus) 和田菁 (Sesbania cannabina $)$ 。种子经消毒 $\left(10 \% \mathrm{H}_{2} \mathrm{O}_{2}\right)$ 后, 直接播在田间。 设置 9 个小区, 小区面积 $10 \mathrm{~m}^{2}(2 \mathrm{~m} \times 5 \mathrm{~m})$, 每个品种 3 个小区(重复), 随机区组排列。播种时, 行距 $40 \mathrm{~cm}$, 株距 $30 \mathrm{~cm}$ 。等幼苗出土 1 周后, 开始间苗, 每小区 留苗 76 株。定期除杂, 不浇水(靠雨水)。待植物进 入现蕾期开始采样分析。因竹豆在重庆地区开花较 迟且少, 采样时只有少数植株有花蕾。

\section{3 蜡质提取}

分别采集每个小区各植物不同植株相同叶位叶 片 5 片, 同时按留茬 $5 \mathrm{~cm}$ 刈割后, 剪取茎段。提蜡 前, 利用 WinFOLIA 专业组织图像分析系统(Regent
Instrument Inc, Canada) 和数字化扫描仪(EPSON V750, Japan)测定茎、叶的正投影面积, 而 3 种豆科 绿肥作物的茎可近似看作圆柱体，故茎、叶面积可 通过以下公式进行计算。

叶片面积 $=2 \times$ 投影面积

茎秆表面积 $=\pi \times$ 投影面积

每个小区叶片和茎秆样品, 在室温条件下分别 用含 $5 \mu \mathrm{g}$ 内标 $(24$ 烷)的氯仿 $(5 \mathrm{~mL})$ 涡旋浸提 3 次, 每 次 $30 \mathrm{~s}$ 。提取液混合后经 $40^{\circ} \mathrm{C}$ 氮气吹干, 加入 $20 \mu \mathrm{L}$ BSTFA [N,O-Bis (三甲基硅烷基)三氟乙酰胺])和 20 $\mu \mathrm{L}$ 吡啶, 于 $70^{\circ} \mathrm{C}$ 衍生反应 $45 \mathrm{~min}$, 多余衍生剂用氮 吹仪再次吹干。将所得提取物溶于 $1 \mathrm{~mL}$ 氯仿, 进行 气相色谱和质谱分析。

\section{4 气相色谱 - 质谱(GC-MS) 分析}

采用 $9790 \mathrm{II}$ 气相色谱仪(浙江福立)分析蜡质含 量。色谱柱为 DM-5 (5\%二苯基 + 95\%二甲基聚硅 氧烷固定液), 毛细管柱长 $30 \mathrm{~m}$, 直径 $0.32 \mathrm{~mm}$, 液 膜厚度 $0.25 \mu \mathrm{m}$ 。氮气作为载气; 进样量为 $1.0 \mu \mathrm{L}$; 柱膜和 FID 检测器的温度分别为 $300^{\circ} \mathrm{C}$ 和 $320^{\circ} \mathrm{C}$; 分 流比为 $10: 3$ 。初始温度为 $80^{\circ} \mathrm{C}$, 以每分钟 $15^{\circ} \mathrm{C}$ 的 速度升温至 $260^{\circ} \mathrm{C}$, 并保持 $10 \mathrm{~min}$, 之后以每分钟 $2^{\circ} \mathrm{C}$ 升温至 $290^{\circ} \mathrm{C}$, 保持 $1 \mathrm{~min}$; 再以每分钟 $5^{\circ} \mathrm{C}$ 的速 度升温至 $320^{\circ} \mathrm{C}$, 并保持 $10 \mathrm{~min}^{\circ}$ 。利用气相色谱一质 谱联用仪(GCMS-AOC-5000 Plus)鉴定蜡质组分, 程 序升温方式同色谱分析。鉴定成分时主要根据离子峰 质荷比 $(\mathrm{m} / \mathrm{z})$ 结合已发表相关成分质谱图, 解析化合物 结构。基于 FID 峰值量化蜡质, 根据质谱所鉴定的标 准确定色谱中各种化学成分的出峰位置, 用内标计算 出植物单位面积的实际蜡质含量, 单位为 $\mu \mathrm{g} \mathrm{cm}^{-2}$ 。

\section{5 数据分析}

试验数据为 3 个重复的平均值 \pm 标准误。采用 SPSS(17.0)统计软件, 利用单因素方差分析 (OneWay ANOVA)比较不同作物茎、叶的蜡质总含量、 蜡质各组分含量及链长分布特征, 显著性检验水平 为 $P=0.05$ (1.s.d.)。

\section{2 结果与分析}

\section{1 蜡质组分鉴定}

以质谱分析获得化合物离子峰质荷比 $(\mathrm{m} / \mathrm{z})$, 再 结合文献中对蜡质成分的鉴定质谱图，从柽麻茎中 鉴定出 5 类物质, 包括脂肪酸、初级醇、醛类、烷 烃和萜类化合物, 叶片中鉴定出 4 类物质, 包括初 级醇、醛类、烷烃和萜类化合物。田菁茎中发现 7 类 
物质, 包括脂肪酸、初级醇、烷基酯、醛类、烷烃、 二醇、萜类和固醇类化合物, 叶片中鉴定出 5 类物质, 包括脂肪酸、初级醇、醛类、烷烃和萜类。其中二醇 为 1,18-30 烷醇和 1,16-30 烷醇, 其中 1,16-30 烷醇离 子峰构成为 $\mathrm{m} / \mathrm{z}$ 73(100\%)、103(38\%)、149(59\%)、 285(23\%)、299(63\%)、313(25\%)、387(8\%)、401(15\%)、 415(6\%)、598(质量峰)(图 1)。竹豆茎和叶中均鉴定出 5 类物质, 包括脂肪酸、初级醇、醛类、烷烃和萜类。 柽麻和田菁茎和叶均有低于 $10 \%$ 左右的成分未能被 鉴定, 而竹豆茎和叶中未鉴定成分高达 $30 \%$ 左右。

\section{2 茎、叶蜡质总量及组分相对含量分析}

柽麻、田菁、竹豆 3 种绿肥作物的茎、叶蜡质总 含量存在显著种间及部位差异, 其中柽麻茎蜡质总含 量为 $16.33 \mu \mathrm{g} \mathrm{cm}^{-2}$, 显著高于田菁茎 $\left(6.45 \mu \mathrm{g} \mathrm{cm}^{-2}\right)$ 和 竹豆茎 $\left(0.72 \mu \mathrm{g} \mathrm{cm}^{-2}\right)$ (图 2)。就茎和叶比较, 柽麻茎显 著高于叶片, 其他 2 种植物茎和叶之间无显著差异。

柽麻茎蜡质中, 烷烃为优势成分, 占蜡质总量 的 $57.38 \%$, 其次是初级醇, 占 $14.23 \%$, 脂肪酸占 $11.23 \%$ ，醛类和萜类化合物均低于 $7 \%$ ，未鉴定蜡质 组分约占 2.96\%（图 3)。叶片中初级醇为优势成分, 占蜡质总量的 $50.12 \%$, 醛类和萜类化合物分别占 $12.77 \%$ 和 $15.95 \%$, 而烷烃比例只有 $5.26 \%$, 约 $12.45 \%$ 的成分未能鉴定出来。
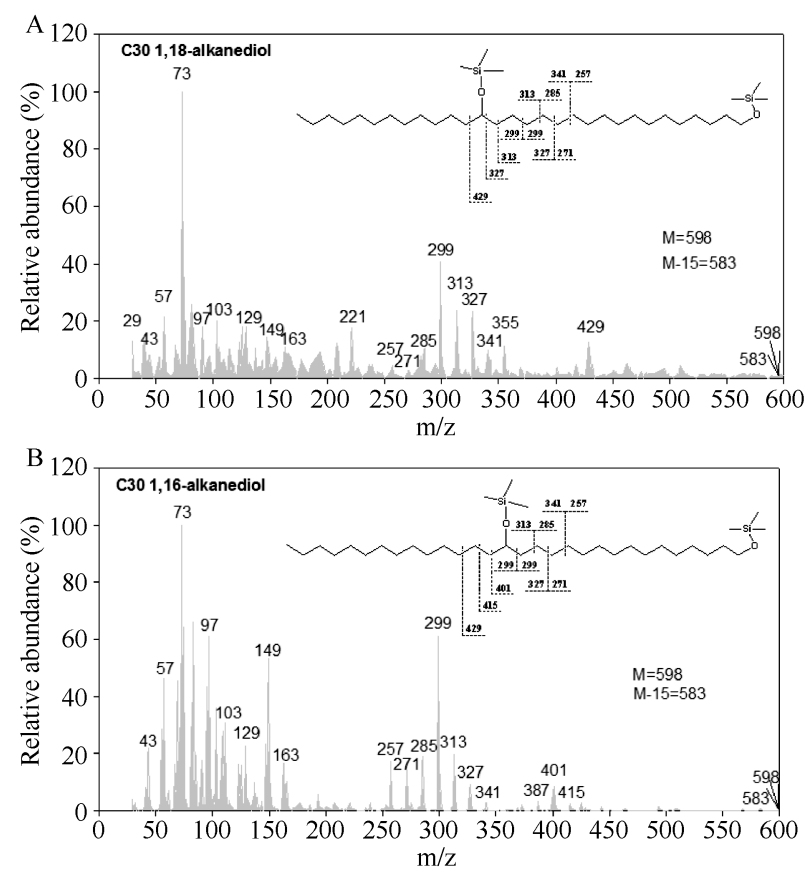

图 1 田菁茎蜡质中双醇化学结构分析

Fig. 1 Chemical structure of diols identified in Sesbania cannabina stem wax

A: 1,18 - 30 双醇; B: 1,16 30-双醇。

A: C30 1,18-alkanediol; B: C30 1,16-alkanediol.
田菁茎、叶蜡质中的优势成分均为初级醇, 其 中茎中占总蜡质的 $30.12 \%$, 而叶中高达 $71.21 \%$ 。其 次是醛类，茎和叶中分别占总蜡质的 $22.89 \%$ 和 $19.39 \%$ (图 4)。茎中发现的二醇, 所占比例为 $3.58 \%$, 烷基酯比例为 $0.44 \%$ 。脂肪酸、烷烃和三萜类物质 在茎和叶中比例分别为 $7.99 \%$ 和 $3.86 \% 、 6.59 \%$ 和 $3.29 \% 、 14.63 \%$ 和 $0.11 \%$ 。另外，茎和叶中均有 $8.55 \%$ 和 $2.13 \%$ 的末知成分。

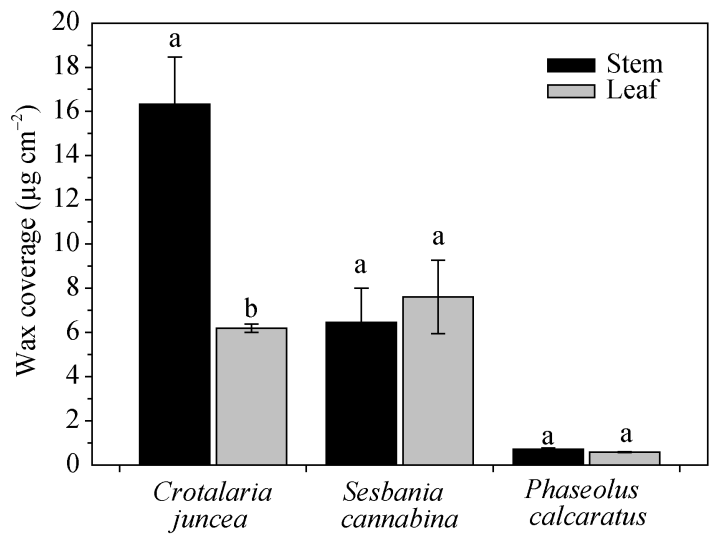

图 2 柽麻、田菁、竹豆的茎和叶蜡质总量

Fig. 2 Wax coverage in stem and leaf of Crotalaria juncea, Sesbania cannabina, and Phaseolus calcaratus 数据柱上不同小写字母表示品种内差异显著 $(P<0.05)$ 。

Different lowercase letters above the data bar within each species represent significant difference at $P<0.05$.

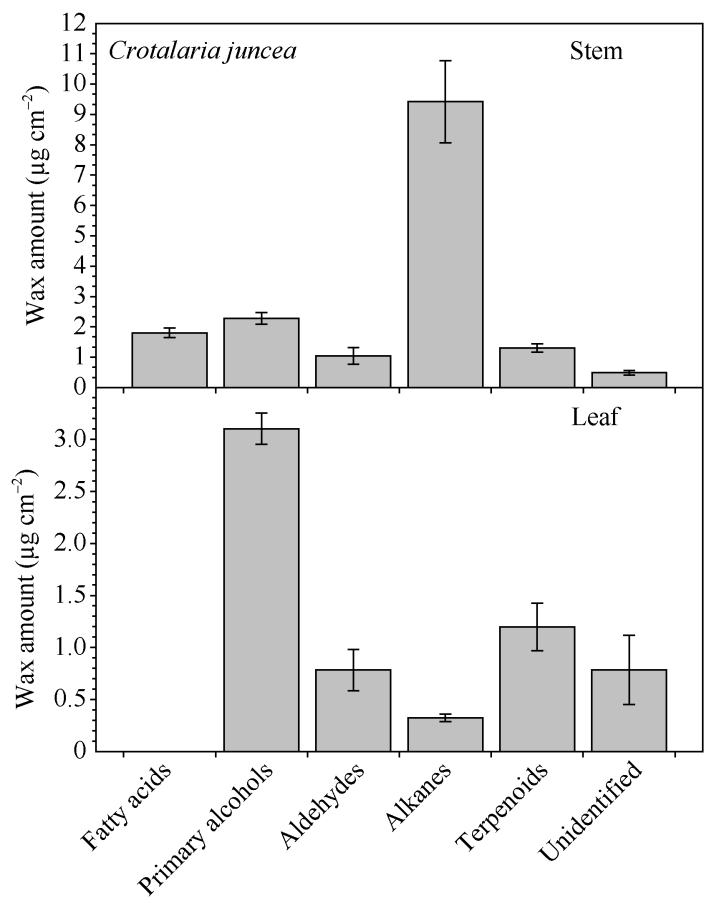

图 3 柽麻茎和叶中主要蜡质组分实际含量

Fig. 3 Amount of wax compound class on stem and leaf of Crotalaria juncea 
竹豆茎、叶蜡质中的优势成分均为烷烃，分别 占总蜡质的 $40.79 \%$ 和 $39.27 \%$ 。其次茎中分别为醛 $(25.28 \%)$ 、初级醇 $(12.18 \%)$ 和三萜类 $(2.61 \%)$ ，叶中三 类组分的比例分别为 $8.34 \% 、 3.75 \%$ 和 $12.69 \%$ (图 5)。 茎和叶中均有小于 $2 \%$ 的脂肪酸, 以及 $17.22 \%$ 和 $22.66 \%$ 的末知成分。

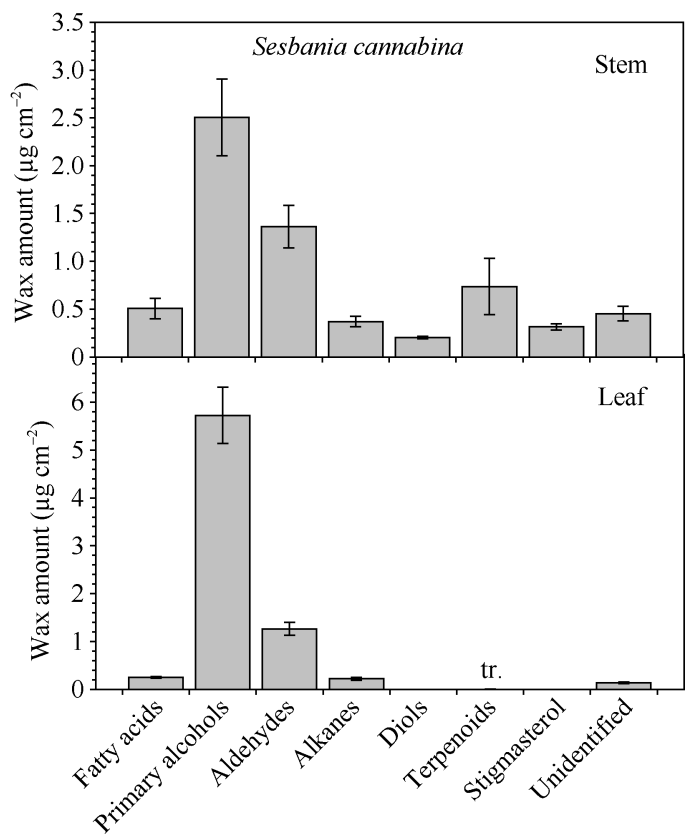

图 4 田菁茎和叶主要蜡质组分实际含量

Fig. 4 Amount of wax compound class on stem and leaf of Sesbania cannabina

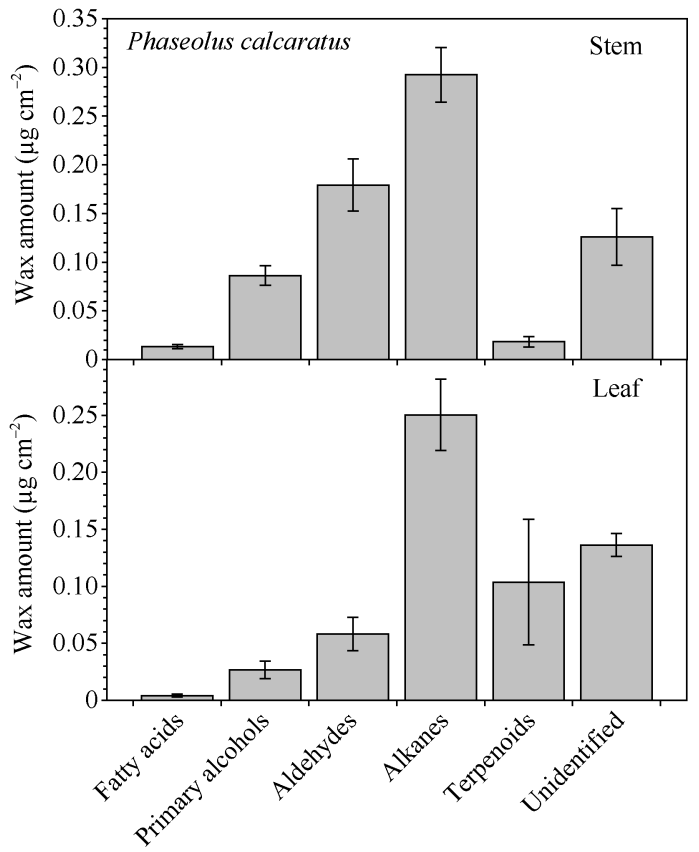

图 5 竹豆茎和叶主要蜡质组分实际含量

Fig. 5 Amount of wax compound class on stem and leaf of Phaseolus calcaratus

\section{3 碳链分布特征分析}

不同绿肥作物中发现的蜡质组分多数以同系物 的形式存在, 如脂肪酸和初级醇均为不同链长的偶 数碳分子, 而醛和烷烃为奇数和偶数碳共存的化合 物, 且烷烃表现为明显的奇数优势, 醛表现为偶数 优势。

2.3.1 柽麻主要蜡质组分碳链分布特征柽麻茎 中脂肪酸碳链分布在 $\mathrm{C}_{20} \sim \mathrm{C}_{32}$ 之间, 其中以 $\mathrm{C}_{30}$ 为优 势组分, 占总脂肪酸的 $33.33 \%$, 其次为 $\mathrm{C}_{32}$, 占 $22.20 \%$ (图 6)。初级醇碳链分布茎中在 $\mathrm{C}_{22} \sim \mathrm{C}_{34}$ 之间, 叶中在 $\mathrm{C}_{24} \sim \mathrm{C}_{32}$ 之间, 二者均以 $\mathrm{C}_{28}$ 为优势组分, 分别 占总初级醇的 $45.34 \%$ 和 $68.66 \%$; 茎中含有较高比例 的 $\mathrm{C}_{32}$, 达到 $31 \%$, 而叶中只有 $0.14 \%$ 。茎和叶中醛的 碳链分布存在较大差异, 茎中以 $\mathrm{C}_{32}$ 为优势组分 $(52.13 \%)$, 叶中以 $\mathrm{C}_{30}$ 为优势组分 $(70.83 \%)$ 。烷烃链长 在 $\mathrm{C}_{25} \sim \mathrm{C}_{33}$ 之间, 茎和叶均以 $\mathrm{C}_{31}$ 为优势组分, 分别占 总烷烃的 $79.49 \%$ 和 $59.56 \%$ 。萜类物质, 茎和叶中均 以羽扇豆醇为最高 $(80 \%$ 左右), 其中茎中还有 $20 \%$ 左 右 $\alpha$-香树脂醇, 叶中有 $15 \%$ 左右的乙酰化羽扇亘醇。

2.3.2 田菁主要蜡质组分碳链分布特征田菁茎 和叶中的脂肪酸碳链分布范围在 $\mathrm{C}_{20} \sim \mathrm{C}_{32}$, 其中茎中 以 $\mathrm{C}_{30}$ 为优势组分 $(68.35 \%)$, 而叶中以 $\mathrm{C}_{26}$ 为优势组分 $(30.15 \%)$ (图 7)。初级醇碳链分布在茎和叶中差异较 大, 其中茎中在 $\mathrm{C}_{26} \sim \mathrm{C}_{32}$, 叶中在 $\mathrm{C}_{22} \sim \mathrm{C}_{32}$, 但 2 个部位 均以 $\mathrm{C}_{30}$ 为优势组分, 分别占 $83.05 \%$ 和 $73.25 \%$ 。醛碳 链分布茎中在 $\mathrm{C}_{28} \sim \mathrm{C}_{34}$, 叶中在 $\mathrm{C}_{28} \sim \mathrm{C}_{32}, 2$ 个部位均以 $\mathrm{C}_{30}$ 为优势组分, 分别占 $46.96 \%$ 和 $52.53 \%$ 。烷碳链分 布茎中在 $\mathrm{C}_{25} \sim \mathrm{C}_{33}$, 叶中在 $\mathrm{C}_{25} \sim \mathrm{C}_{31}, 2$ 个部位均以 $\mathrm{C}_{29}$ 为优势组分，分别占 $41.77 \%$ 和 $51.18 \%$ 。茎中所鉴定的 二醇为 $\mathrm{C}_{30}$ 同分异构体，其中 1,18-30 烷醇占 $27.91 \%$, 1,16-30 烷醇占 $72.09 \%$ 。茎中发现的萜类物质为 $\beta$-香 树脂醇和羽扇豆醇，而叶中只有 $\beta$-香树脂醇。

2.3.3 竹豆主要蜡质组分碳链分布特征竹豆茎 和叶中脂肪酸碳链分布均在 $\mathrm{C}_{22} \sim \mathrm{C}_{30}$ 之间, 且均以 $\mathrm{C}_{28}$ 为优势组分 $(60 \%$ 左右)(图 8$)$ 。初级醇碳链分布, 茎中在 $\mathrm{C}_{24} \sim \mathrm{C}_{30}$, 叶中在 $\mathrm{C}_{24} \sim \mathrm{C}_{34}$, 其中茎中以 $\mathrm{C}_{28}$ 为优 势组分 $(68.75 \%)$, 叶中以 $\mathrm{C}_{32}$ 为优势组分 $(60.38 \%)$ 。茎 中醛碳链在 $\mathrm{C}_{28} \sim \mathrm{C}_{32}$, 其中 $\mathrm{C}_{30}$ 为优势组分 $(88.75 \%)$, 而叶中只有 $\mathrm{C}_{28}$ 醛。茎和叶中烷烃碳链分布均在 $\mathrm{C}_{25}$ $\mathrm{C}_{33}$ 之间, 且均以 $\mathrm{C}_{31}$ 为优势组分, 分别占 $40.75 \%$ 和 $60.26 \%$ 。茎和叶中的萜类物质均为 $\beta$-香树脂醇和羽 扇豆醇, 其中茎以 $\beta$-香树脂醇为优势组分 $(73.69 \%)$, 叶以羽扇豆醇为优势组分 $(72.97 \%)$ 。 

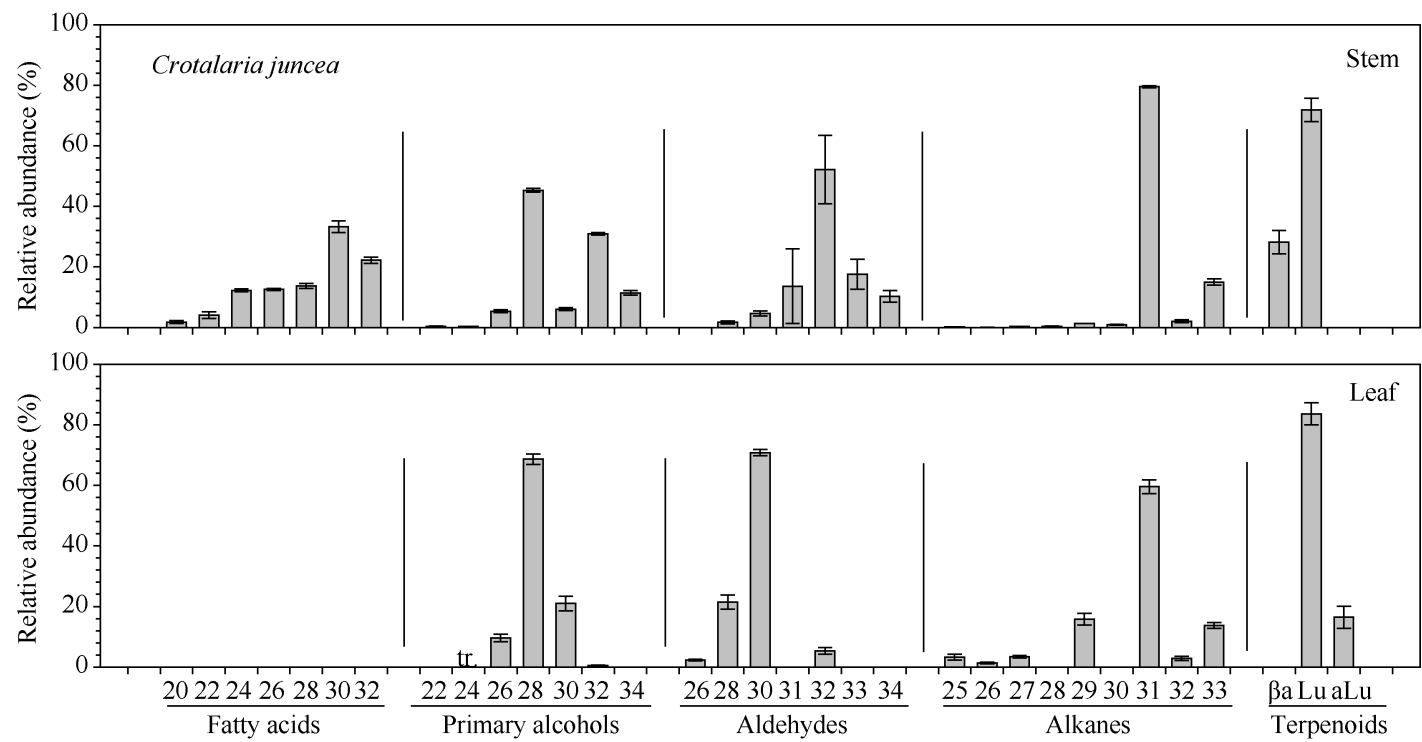

图 6 柽麻茎和叶蜡质各组分同系物相对含量

Fig. 6 Relative abundance of compound homologs in Crotalaria juncea

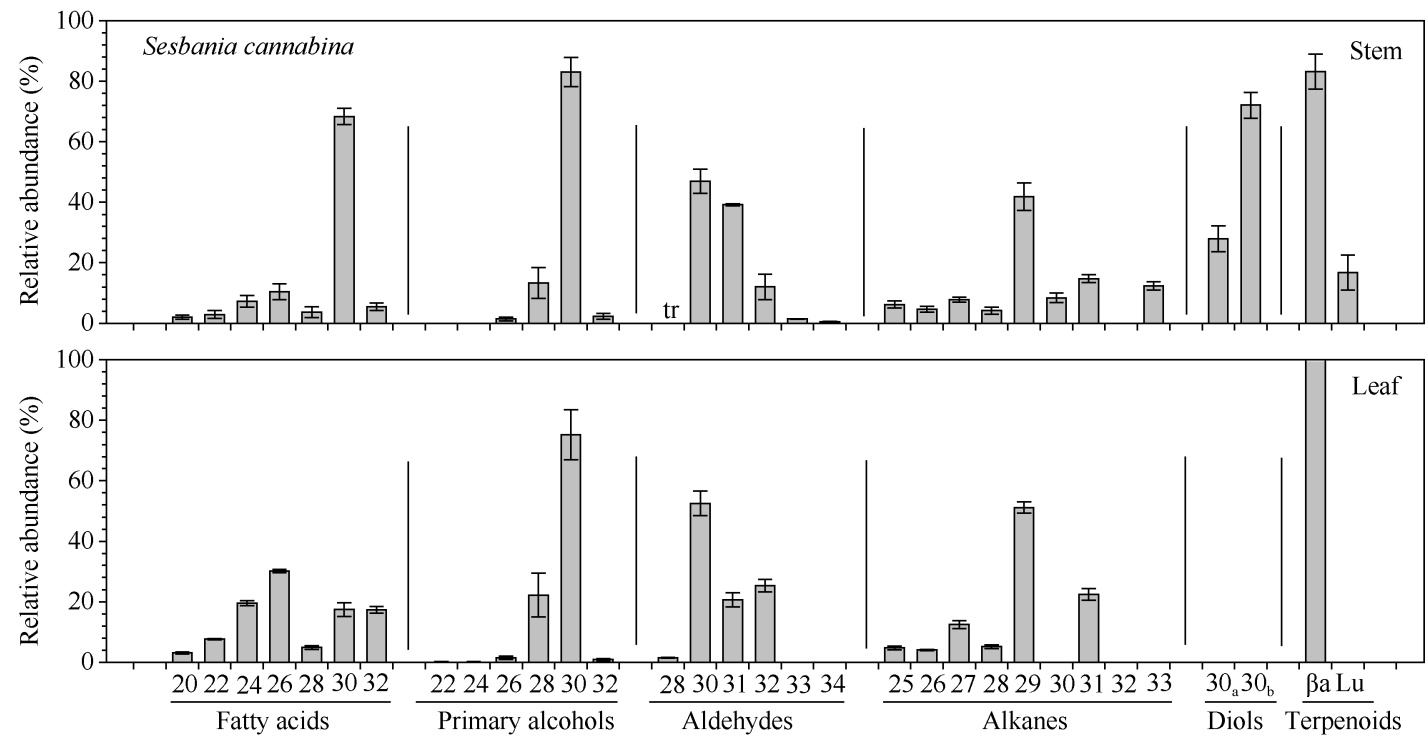

图 7 田菁茎和叶蜡质各组分同系物相对含量

Fig. 7 Relative abundance of compound homologs in Sesbania cannabina

\section{3 讨论}

3 种豆科绿肥作物的茎和叶中均含有脂肪酸、初 级醇、醛类和烷烃, 且这些组分含量总和占总蜡质含 量的比例超过 $60 \%$ 。说明烷合成途径和醇合成途径是 这些植物合成角质层蜡质的主要途径。这与在其他主要 作物上的发现是一致的 ${ }^{[9]}$ 。然而, 䓲类植物叶角质层蜡 质以烷基酯为优势成分 ${ }^{[19]}$, 羊草以二酮为优势成分 ${ }^{[10]}$ 。 这些结果暗示了, 在长期进化过程中, 不同植物为适 应不同的生存环境其角质层蜡质生物合成产生了不同 的进化方向。此外, 我们还发现田菁茎和叶中的蜡质
组分存在较大差异, 有些成分只出现在叶片, 如二醇 和固醇类物质, 其中二醇为 1,18-30 烷醇和 1,16-30 烷 醇。在高山罂粟(Papaver alpinum)叶片中也鉴定出 C-30 的二醇同系物, 但其羟基位置在 $1,11^{[20]}$, 在蓄麻 (Ricinus communis)叶片中, 鉴定出链长 C-22 到 C-28、 羟基位置在 1,3 的二醇同系物 ${ }^{[21]}$ 。Busta 和 Jetter ${ }^{[22]}$ 认 为这些碳链一头为初级醇、碳链中间存在另一个羟基 的二元醇结构, 其生物合成过程可能是由 P450 加氧酶 参与完成的。在拟南芥 $(A$. thaliana $)$ 中, MAH1 作为羟 化酶参与了二醇的合成 ${ }^{[23]}$ 。田菁叶中二醇物质的发现, 为今后研究二醇生物合成途径提供了新的材料。 

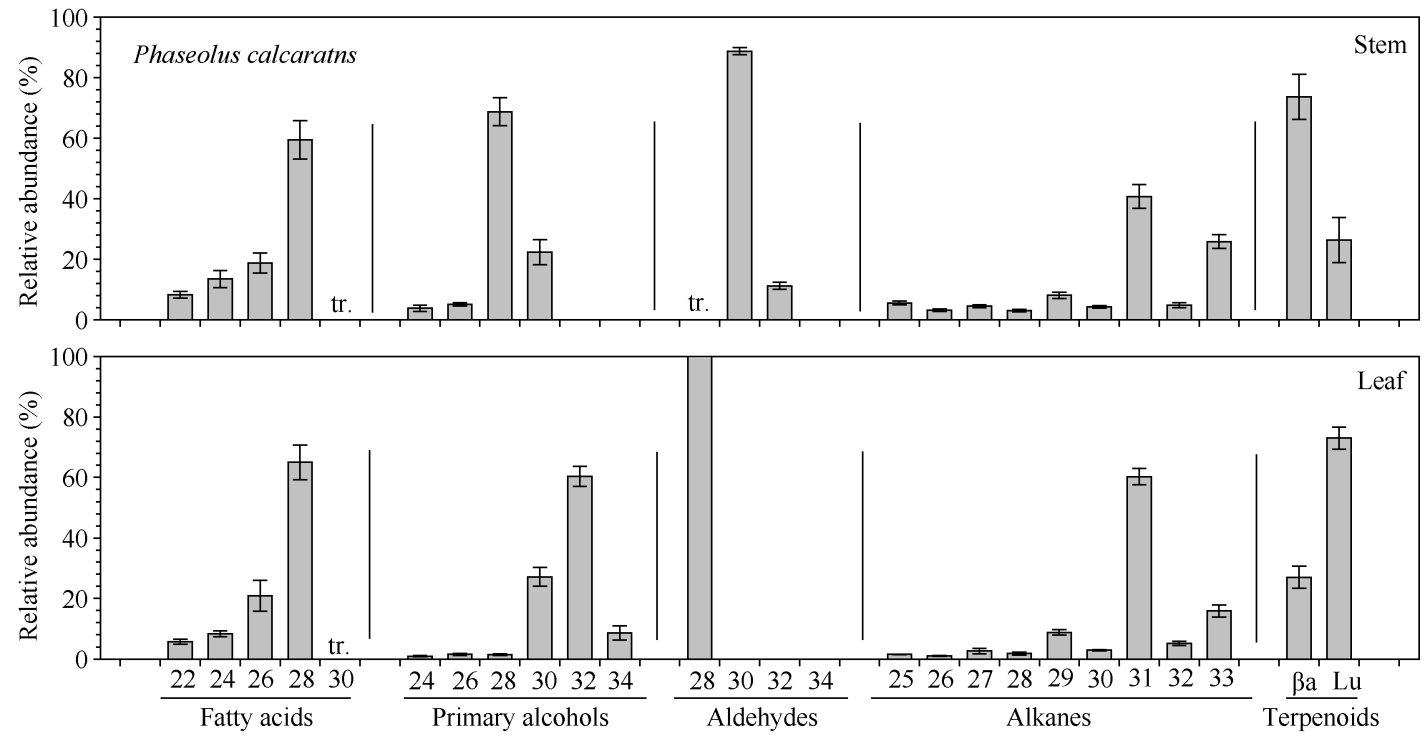

图 8 竹豆茎和叶蜡质各组分同系物相对含量

Fig. 8 Relative abundance of compound homologs in Phaseolus calcaratus

3 种豆科绿肥作物茎和叶中蜡质含量存在较大 差异, 其中茎蜡质含量最高的是柽麻, 其次是田菁, 竹豆蜡质含量最低。一般情况下，植物蜡质含量的 增加与其抗旱性的提高相联系 ${ }^{[2]}$ 。尽管都是夏季绿 肥作物, 蜡质含量较低的竹豆为藤本植物, 群体高 度低、叶片数量多、地表覆盖度高, 在进化过程中 其生长环境可能相对湿润, 不需要过高的蜡质沉积 就能适应生长环境。而柽麻与田菁株型直立, 地表 覆盖度低, 生长环境相对干燥, 叶片可能需要沉积 较多的蜡质来抵御逆境胁迫。当然, 影响植物抗逆 性的因素较多, 今后有必要进一步分析几种植物蜡 质含量与其抗旱性的关系。就茎和叶角质层蜡质含 量比较, 拟南芥茎秆高于叶片 ${ }^{[24]}$, 马铃薯 (Solanum tuberosum) 无显著差异 ${ }^{[25]}$, 蒲公英(Taraxacum officinale)叶片高于茎秆 ${ }^{[26]}$, 本试验中柽麻茎高于叶片, 而田菁和竹豆茎和叶蜡质无显著差异。这些结果说 明不同植物茎和叶角质层蜡质的沉积存在器官特异 性, 且这可能与各自不同的生理功能有关。对 12 个 大麦品种的比较发现, 蜡质沉积较多的品种具有较 高的抗旱性 ${ }^{[3]}$ 。转录因子MYB96在亚麻荠(Camelina sativa) 中的超量表达可显著增加蜡质沉积, 并提高 植物抗旱性 ${ }^{[27]}$ 。

在茎和叶蜡质组分方面, 柽麻茎以烷烃为优势 组分, 而叶以初级醇为优势组分, 说明参与蜡质合 成的主效基因在 2 个器官的表达存在差异, 如拟南 芥参与初级醇合成的CER4在叶中较高表达, 而参与 烷合成的CER1在茎中高表达 ${ }^{[28]}$ 。田菁茎和叶中的优
势组分均为初级醇 $(30.12 \%$ 和 $71.21 \%)$ ，且烷烃组分 比例不足 $10 \%$, 说明田菁角质层蜡质以醇合成途径 为主。竹豆茎和叶中的优势成分均为烷烃, 说明其 角质层蜡质合成以烷合成途径为主。在其他作物上 也有类似的研究结果, 如紫花苜宿叶以初级醇为主, 茎以烷为主 ${ }^{[14]}$; 亚麻荠 (C. sativa) 叶以烷基酯为主, 茎以三萜类化合物为主 ${ }^{[29]}$; 而玉米叶以初级醇为主, 茎以醛为主 ${ }^{[30]}$ 。尽管目前尚无法确定这些优势组分 是否在植物抗逆方面起主要作用, 然而干旱胁迫下 烷类含量的增加被认为与多数植物的抗旱性提高有 关 ${ }^{[2]}$; 植物叶表长链醛类的缺失可抑制病原真菌孢 子的萌发 ${ }^{[31]}$ 。

3 种绿肥作物茎和叶中主要的蜡质组分均以同 系物形式存在, 且整体表现出脂肪酸、初级醇和醛以 偶数碳占优势、而烷烃以奇数碳占优势的碳链分布特 征。这与绝大多数植物上的研究结果是一致的 ${ }^{[32]}$ 。就 蜡质组分内同系物优势化合物碳链长度分析, 柽麻 和竹豆茎和叶均以 $\mathrm{C}_{31}$ 为优势化合物, 而田菁以 $\mathrm{C}_{29}$ 为优势化合物。说明参与烷烃生物合成的同源基因 在茎和叶中是一致的。茎和叶初级醇优势化合物在 柽麻和田菁是一致的, 分别为 $\mathrm{C}_{28}$ 和 $\mathrm{C}_{30}$; 而竹豆茎中 以 $\mathrm{C}_{28}$ 为优势化合物, 叶中以 $\mathrm{C}_{32}$ 为优势化合物。出现 这一结果的原因可能在于链长延长酶(FAE)及参与 初级醇合成的CER4在竹豆不同器官存在差异 ${ }^{[33]}$ 。醛 被认为是烷合成途径和醇合成途径的中间产物 ${ }^{[9]}$ 。 几种绿肥作物茎和叶中醛的优势化合物存在较大差 异, 暗示不同器官的醛可能来自不同的合成途径。 


\section{4 结论}

从 3 种夏季绿肥作物茎和叶中共鉴定出 8 类化 合物, 包括脂肪酸、初级醇、烷基酯、醛类、烷烃、 二醇、萜类和固醇类化合物。尽管主要蜡质组分在 品种及部位间一致，但不同作物及部位又有各自独 特的组分存在, 如柽麻茎中未检测出脂肪酸, 田菁 茎中鉴定出固醇类和二醇化合物。其中田菁茎中的 二醇化合物初步结构解析为 1,18-30 烷醇和 1,16-30 烷醇。此外, 不同物种、不同部位所积累的优势组 分及优势化合物也存在一定差异, 说明参与蜡质合 成的基因在物种、器官间有所不同。这为今后从分 子水平上揭示角质层蜡质参与夏季绿肥作物抗逆机 制提供了理论基础。

\section{References}

[1] N'Dayegamiye A, Tran T S. Effects of green manures on soil organic matter and wheat yields and N nutrition. Can J Soil Sci, 2001, 81: 371-382.

[2] Yeats T H, Rose J K C. The formation and function of plant cuticles. Plant Physiol, 2013, 163: 5-20.

[3] Gonzalez A, Ayerbe L. Effect of terminal water stress on leaf epicuticular wax load, residual transpiration and grain yield in barley. Euphytica, 2010, 172: 341-349.

[4] Skorska E, Szwarc W. Influence of UV-B radiation on young triticale plants with different wax cover. Biol Planta, 2007, 51: 189-192.

[5] Zhu M, Riederer M, Hildebrandt U. Very-long-chain aldehydes induce appressorium formation in ascospores of the wheat powdery mildew fungus Blumeria graminis. Fungal Biol, 2017, 121: 716-728.

[6] 朱命炜, 王红星, 李建军, 李俊英, 王太霞, 李景原. 木立芦 荟发育过程中叶表皮角质膜和蜡质的变化. 电子显微学报, 2004, 23: 670-673.

Zhu M W, Wang H X, Li J J, Li J Y, Wang T X, Li J Y. Changes in the cuticle and wax of the leaves during the development of aloe (Aloe arborescens Mill.). J Chin Elect Microscopy Soc, 2004, 23: 670-673 (in Chinese with English abstract).

[7] 韦存虚, 王建波, 陈义芳, 周卫东, 孙国荣. 盐生植物星星草 叶表皮具有泌盐功能的蜡质层. 生态学报, 2004, 24: 2451-2456.

Wei C X, Wang J B, Cheng Y F, Zhou W D, Sun G R. Epicuticular wax of leaf epidermis: a functional structure for salt excretion in a halophyte Puccinellia tenuiflora. Acta Ecol Sin, 2004, 24: 2451-2456 (in Chinese with English abstract)

[8] Jetter R, Riederer M. Composition of cuticular waxes on $O s$ munda regalis Fronds. J Chem Ecol, 2000, 26: 399-412.

[9] Lee S B, Suh M C. Advances in the understanding of cuticular waxes in Arabidopsis thaliana and crop species. Plant Cell Rep, 2015, 34: 557.

[10] 李晓婷, 赵晓, 王登科, 黄蕾, 姚露花, 王党军, 和玉吉, 郭彦 军. 天然草地植物叶角质层蜡质的化学组成及其对自由放牧
的响应. 草业学报, 2018, 27(6): 137-147.

Li X T, Zhao X, Wang D K, Huang L, Yao L H, Wang D J, He Y J, Guo Y J. Chemical profiles of cuticular waxes in arid steppe plant species and their responses to continuous grazing. Acta Pratacult Sin, 2018, 27(6): 137-147 (in Chinese with English abstract).

[11] Bernard A, Joubès J. Arabidopsis cuticular waxes: advances in synthesis, export and regulation. Prog Lipid Res, 2013, 52: 110-129.

[12] Kunst L, Samuels L. Plant cuticles shine: advances in wax biosynthesis and export. Curr Opin Plant Biol, 2009, 12: 721-727.

[13] Kosma D K, Rowland O. Answering a four decade-old question on epicuticular wax biosynthesis. J Exp Bot, 2016, 67: 2538-2540.

[14] Zhang J Y, Broeckling C D, Blancaflor E B, Sledge M K, Sumner L W, Wang Z Y. Overexpression of WXP1, a putative Medicago truncatula AP2 domain-containing transcription factor gene, increases cuticular wax accumulation and enhances drought tolerance in transgenic alfalfa (Medicago sativa). Plant J, 2005, 42: 689-707.

[15] Zhou L Y, Ni E D, Yang J W, Zhou H, Liang H, Li J, Jiang D G, Wang Z H, Liu Z L, Zhuang C X. Rice OsGL1-6 is involved in leaf cuticular wax accumulation and drought resistance. PLoS One, 2013, 8: 12.

[16] 杜青峰, 王党军, 于翔宇, 姚露花, 和玉吉, 王瑞, 马生兰, 郭 彦军. 玉米间作夏季绿肥对当季植物养分吸收和土壤养分有 效性的影响. 草业学报, 2016, 25(3): 225-233.

Du Q F, Wang D J, Yu X Y, Yao L H, He Y J, Wang R, Ma S L, Guo Y J. The effects of corn and green manure intercropping on soil nutrient availability and plant nutrient uptake. Acta Pratac Sin, 2016, 25(3): 225-233 (in Chinese with English abstract)

[17] 张国发, 吴园园, 徐太海, 梁彦涛. 田菁秸秆还田对松嫩平原 盐碱土改良效果的研究. 大庆师范学院学报, 2018, 38(3): 48-50.

Zhang G F, Wu Y Y, Xu T H, Liang Y T. The effects of returning Sesbania cannabina straw on improving soil quality in alkalized soils of Songnen Plain. J Daqing Norm Univ, 2018, 38(3): 48-50 (in Chinese).

[18] 杨湘如, 郑永发. 幼龄果园套种竹豆生态效益试验研究. 中国 水土保持, 1991, (11): 29-30.

Yang X R, Zheng Y F. The ecological profits of Phaseolus calcaratns intercropping with young orchard. Chin Water Soil Conserv, 1991, (11): 29-30 (in Chinese).

[19] Guo Y, Li J J, Busta L, Jetter R. Coverage and composition of cuticular waxes on the fronds of the temperate ferns Pteridium aquilinum, Cryptogramma crispa, Polypodium glycyrrhiza, Polystichum munitum and Gymnocarpium dryopteris. Ann Bot, 2018, 122: 555-568.

[20] Jetter R, Riederer M, Seyer A, Mioskowski C. Homologous long-chain alkanediols from Papaver leaf cuticular waxes. Phytochemistry, 1996, 42: 1617-1620.

[21] Vermeer C P, Nastold P, Jetter R. Homologous very-long-chain 1,3-alkanediols and 3-hydroxyaldehydes in leaf cuticular waxes of Ricinus communis L. Phytochemistry, 2003, 62: 433-438.

[22] Busta L, Jetter R. Moving beyond the ubiquitous: the diversity and biosynthesis of specialty compounds in plant cuticular waxes. Phytochem Rev, 2018, 17: 1275-1304. 
[23] Wen M, Jetter R. Composition of secondary alcohols, ketones, alkanediols, and ketols in Arabidopsis thaliana cuticular waxes. $J$ Exp Bot, 2009, 60: 1811-1821.

[24] Hegebarth D, Buschhaus C, Wu M, Bird D, Jetter R. The composition of surface wax on trichomes of Arabidopsis thaliana differs from wax on other epidermal cells. Plant J, 2016, 88: 762-774.

[25] Guo Y J, Jetter R. Comparative analyses of cuticular waxes on various organs of potato (Solanum tuberosum L.). J Agric Food Chem, 2017, 65: 3926-3933.

[26] Guo Y J, Busta L, Jetter R. Cuticular wax coverage and composition differ among organs of Taraxacum officinale. Plant Physiol Biochem, 2017, 115: 372-379.

[27] Lee S, Kim H, Kim R, Suh M. Overexpression of Arabidopsis MYB96 confers drought resistance in Camelina sativa via cuticular wax accumulation. Plant Cell Rep, 2014, 33: 1535-1546.

[28] Rowland O, Zheng H, Hepworth S R, Lam P, Jetter R, Kunst L. CER4 encodes an alcohol-forming fatty acyl-coenzyme a reductase involved in cuticular wax production in Arabidopsis. Plant Physiol, 2006, 142: 866-877.

[29] Razeq F M, Kosma D K, Rowland O, Molina I. Extracellular lipids of Camelina sativa: characterization of chloroformextractable waxes from aerial and subterranean surfaces. Phytochemistry, 2014, 106: 188-196.

[30] Javelle M, Vernoud V, Depege-Fargeix N, Arnould C, Oursel D, Domergue F, Sarda X, Rogowsky P M. Overexpression of the epidermis-specific homeodomain-leucine zipper IV transcription factor Outer Cell Layer1 in maize identifies target genes involved in lipid metabolism and cuticle biosynthesis. Plant Physiol, 2010, 154: 273-286.

[31] Hansjakob A, Riederer M, Hildebrandt U. Appressorium morphogenesis and cell cycle progression are linked in the grass powdery mildew fungus Blumeria graminis. Fungal Biol, 2012, 116: 890-901.

[32] Jetter R, Kunst L, Samuels A L. Composition of plant cuticular waxes. In: Riederer C. Muller, eds. Biology of the Plant Cuticle. Oxford: Blackwell Publishing Ltd, 2006. pp 145-181.

[33] Haslam T M, Mañas-Fernández A, Zhao L, Kunst L. Arabidopsis ECERIFERUM2 is a component of the fatty acid elongation machinery required for fatty acid extension to exceptional lengths. Plant Physiol, 2012, 160: 1164-1174. 